\title{
La tutoria entre pares: una estrategia de enseñanza y aprendizaje de histología en la Universidad Industrial de Santander
}

\author{
Daniel Mauricio Torrado-Arenas* \\ Edgar Fabián Manrique-Hernández** \\ Jaime Otoniel Ayala-Pimentel ${ }^{* * *}$
}

\begin{abstract}
* Médico y cirujano. Miembro de la Sociedad Estudiantil de Investigación Médica. Auxiliar académico del Programa de Asesoría para el Mejoramiento del Rendimiento Académico en Morfofisiología General. Universidad Industrial Santander. Bucaramanga. Santander. Colombia.

**Estudiante de VI año de medicina. Miembro de la Sociedad Estudiantil de Investigación Médica. Universidad Industrial de Santander. Bucaramanga. Santander. Colombia.

***Médico y cirujano. Magister en Morfología. Especialista en Docencia Universitaria. Profesor titular. Departamento ciencias básicas. Escuela de Medicina grupo de Neurociencias y Comportamiento UIS-UPB. Universidad Industrial Santander. Bucaramanga. Santander. Colombia.

Correspondencia: Dr. Daniel Torrado Arenas. Dirección: Carrera 29 No. 96-78, Diamante I. Bucaramanga. Santander. Colombia. Teléfono: (+57) 316 301 7593. Correo electrónico: torradoarenasdanielmauricio@hotmail.com
\end{abstract}

\section{RESUMEN}

La tutoría entre pares es una estrategia pedagógica donde estudiantes, generalmente de semestres más avanzados, acompañan a sus pares de semestres inferiores en el refuerzo de los procesos de aprendizaje dentro de un área disciplinar. En la Universidad Industrial de Santander se implementa esta modalidad a través del Programa de Asesoría para el Mejoramiento del Rendimiento Académico, en búsqueda de disminuir la deserción en todos los programas académicos. El objetivo de este trabajo es evidenciar el proceso de las tutorías entre pares mostrando su importancia y describiendo la metodología impartida en la sección de histología de la asignatura morfofisiología y del programa de medicina. Debido a que la tutoría entre pares tiene beneficios tanto para el tutor como el beneficiario, es importante implementar esta estrategia en la transición de la educación básica a la superior y es por ello que el programa se encarga del reclutamiento, capacitación y evaluación de los tutores. Las tutorías se realizan en pequeños grupos dentro del laboratorio con visualización de placas histológicas y usando medios audiovisuales para la descripción de las diferentes estructuras microscópicas. MÉD UIS. 2016;29(1):71-5.

Palabras clave: Tutorial interactivo. Programas. Histología. Educación. Mentores.

\section{Peer tutoring: a strategy of teaching and learning of histology at Universidad Industrial de Santander}

\section{ABSTRACT}

The peer tutoring is a teaching strategy where generally more advanced semesters students, accompanying their peers from lower semesters in reinforcing learning processes within a subject area. In Universidad Industrial de Santander this mode is implemented through "Programa de Asesoría para el Mejoramiento del Rendimiento Académico", seeking to reduce the dropout rate in all academic programs. The objetive of this paper is to reflect on the importance of peer tutoring and describe the methodology provided by the program in the section histology of general morphophysiology land subject from the medicine program. Because peer tutoring has benefits for both the tutor and the beneficiary, it is important to implement this strategy in the transition from primary school to college and that is why the program recruitment, training and assessment tutors. Tutorials are conducted in small groups in the laboratory with visualization of histological slides and using media to the description of the different microscopic structures. MÉD UIS. 2016;29(1):71-5.

Keywords: Interactive Tutorial. Programs. Histology. Education. Mentors.

¿Cómo citar este artículo?: Torrado-Arenas DM, Manrique-Hernández EF, Ayala-Pimentel JO. La tutoría entre pares: una estrategia de enseñanza y aprendizaje de histología en la Universidad Industrial de Santander. MÉD.UIS. 2016;29(1)71-5. 


\section{INTRODUCCIÓN}

La tutoría entre pares o iguales es una modalidad organizativa y una estrategia pedagógica en la que estudiantes, generalmente de semestres más avanzados, acompañan a sus pares (estudiantes semejantes) en la orientación y el refuerzo de los procesos de aprendizaje dentro de un área disciplinar, que se realiza en pequeños grupos o en parejas ${ }^{1,2}$. Esta estrategia pedagógica tiene beneficios tanto para el tutor como para el tutorado: los beneficiarios refuerzan hábitos de estudio, mejoran el rendimiento académico, adquieren motivación para estudiar y aumentan promedio de calificaciones; y al mismo tiempo, los tutores se favorecen al afianzar sus conocimientos, adquirir valores de solidaridad entre pares, adoptar habilidades de comunicación y obtener experiencia docente 3 .

Se han realizado varios estudios acerca de la utilidad de la estrategia tutor-par, entre ellos la revisión literaria de Santee $J$ et al. quienes encuentran, en la mayoría de los trabajos, un resultado positivo en el rendimiento académico y mayor motivación para estudiar de los alumnos que recibieron tutoría entre pares al compararlos con lo que no la recibieron ${ }^{4,5}$.

También se ha estudiado el efecto de las tutorías en las puntuaciones de las evaluaciones. En el estudio de Dejano $T$ et al, en el cual se examinaron 447 registros de estudiantes de medicina entre los años 1993 y 2000, se analizaron las características de la tutoría en un programa de educación de la Universidad de Brasilia, y encontraron una asociación entre el número de sesiones de tutorías y un aumento en el promedio de las calificaciones finales respecto a las iniciales ${ }^{6}$.

La figura de la tutoría entre pares se desarrolló en el año 2000 en Europa con el surgimiento del Espacio Europeo de Educación Superior (EEES)1. A pesar de ello históricamente hay evidencia de esta práctica en la antigua Grecia. En la actualidad, los métodos de tutoría entre pares han sido explorados en la educación del cuidado de la salud por más de 30 años.

Según la información registrada en el Sistema para la Prevención y de la Deserción en las Instituciones de Educación Superior (SPDIES) para el año 2013, la deserción universitaria en Colombia fue del $44,9 \%$, es decir que uno de cada dos estudiantes que ingresaba a educación superior no culminaba sus estudios. A nivel departamental, Santander tiene el $43 \%$ estando por debajo del referente nacional. Sin embargo al reportar por áreas o programas académicos, las ciencias de la salud con $39,4 \%$ ocupan las menores tasas y para el caso de medicina es de $34,1 \%$.

Por lo anterior, y con el fin de disminuir los índices de deserción y permanecía universitaria, la generación de afecciones de salud física y la mental por reprobación y reincidencia de asignaturas y falta de adaptación al sistema de educación universitario ${ }^{9,10}$, la Universidad Industrial de Santander (UIS) generó el Programa de Asesoría para el Mejoramiento del Rendimiento Académico (PAMRA).

EI PAMRA está basado en la estrategia de tutoría entre pares y se encarga de capacitar a estudiantes como tutores con calidad de auxiliares docentes; estos apoyan los procesos de aprendizaje de asignaturas problemáticas, es decir las de mayor reprobación de todos los programas académicos de la universidad como morfofisiología que es aquella que estudia la composición, función, estructura y características de los tejidos de los seres vivos ${ }^{11,12}$.

PAMRA cuenta con el respaldo de la dirección universitaria, es liderado por profesionales de trabajo social principalmente adscritos a la división de bienestar universitario, dependencia de la vicerrectoría administrativa. Este proyecto se inició como una prueba piloto en 1992, pero solo hasta en 1995 comenzó a prestar su servicio son 91 tutores que atendieron a 639 beneficiarios especialmente del ciclo básico. De los programas académicos de ingenierías en el año 2009, PAMRA contó con la participación de 160 tutores que habían extendido sus servicios a 1000 estudiantes de la mayoria de los programas académicos de la universidad lo que contribuyó a disminuir las cifras de deserción universitaria.

Este tipo de metodología de enseñanza-aprendizaje en el área de la anatomía no ha sido documentada, por lo que el objetivo del presente trabajo es evidenciar el proceso de las tutorias entre pares mostrando su importancia y describiendo la metodología impartida en la sección de histología de las asignaturas morfofisiología y del programa de medicina de la Universidad Industrial de Santander.

\section{Beneficiarios}

El beneficiario es un estudiante que busca ayuda al programa de PAMRA, debido a que la sección 


\section{ERERO-HBRIL}

de histología implica una metodología práctica de técnicas de microscopía óptica, la cual puede resultar nueva en la transición de educación básica del colegio a la educación superior en el ámbito universitario, donde se pueden tener diferentes estilos de aprendizaje ${ }^{2}$. Así, el beneficiario al recibir esta estrategia refuerza sus conocimientos, obtiene motivación y adquiere un hábito de estudio más dirigido al tema, debido al gran contenido que se manejan en este tipo de asignaturas de los programas de salud ${ }^{13}$. La tutoría entre pares favorece la identificación de dificultades y errores del estudiante, ayuda a superar obstáculos del aprendizaje y observa el progreso del beneficiario, interviniendo en oportunamente en asuntos de índole formativa.

\section{$\underline{\text { TUTORES }}$}

El tutor es un estudiante que generalmente se encuentra en semestre avanzado, conocedor de un área específica. Quien al aplicar aprendizaje colaborativo con sus beneficiarios fortalece el conocimiento previo y al estar comprometido con la formación de sus compañeros hace un aporte pedagógico y social hacia los estudiantes. Por lo tanto, ser tutor es una oportunidad para compartir y construir valores como la solidaridad.

Además, otra cualidad que adquiere el que dirige la tutoría es el desarrollo de habilidades de comunicación e interacción social como hablar en público sin timidez y poseer adecuada presentación personal ${ }^{1,14}$. Incluso algunos programas no solo consideran importante aplicar las tutorías en las ciencias básicas, sino en ciencias clínicas, pues como lo demuestran Buckley $S$ et al, esta estrategia repercute en dos áreas importantes; primero, mejora la confianza al hablar con grupos, y en segundo lugar, permite una buena entrevista durante la anamnesis del paciente, al llevarlo a la práctica en clínica ${ }^{15,16}$. Así la tutoría entre pares permite el desarrollo de competencias comunicativas fomentando la capacidad de escucha y argumentación.

Finalmente, un estudiante de medicina formado integralmente debe adquirir habilidades clínicas e investigativas. Por lo descrito como aporte importante que los programas de tutorías entre pares permitan adquirir experiencia en docencia, sobre todo si el tutor planea ser profesor en el área de la salud en un futuro ${ }^{17-9}$.

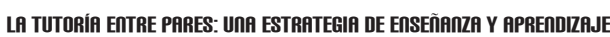
DE HISTOLOGí En LA UחIVERSIDAD InDUSTRIAL DE SATTAกDER

\section{TUTORIAS DE HISTOLOGÍA EN LA UIS}

La UIS abre sus convocatorias para que los estudiantes de mejor rendimiento académico se vinculen como tutores voluntarios por un periodo de un año. Una vez se cumpla el tiempo previsto, se puede ser nombrado como auxiliar docente. Para recibir el título debe cumplir los siguientes requisitos según lo contemplado dentro de la misión del programa PAMRA: tener un promedio igual a mayor a 3,5 (teniendo en cuenta calificaciones de o a 5), estar cursando un semestre igual o mayor a quinto nivel y cumplir con cualidades humanas tales como motivación, responsabilidad, compromiso, respeto, honestidad, justicia, lealtad y tolerancia ${ }^{20}$.

Durante el proceso la universidad capacita a los tutores con talleres mensuales de herramientas pedagógicas orientados por profesionales, una convivencia con jornada pedagógica y recreativa, reuniones mensuales de actualización del programa y evaluación del desempeño por trabajo social. Además, los estudiantes tutores pueden realizar un curso de preparación pedagógica para realizar tutorías, dirigido por profesores de la Red Innova Cesal adscritos a la escuela de tutores del Sistema de Excelencia Académica de la UIS (Ver Figura 1).

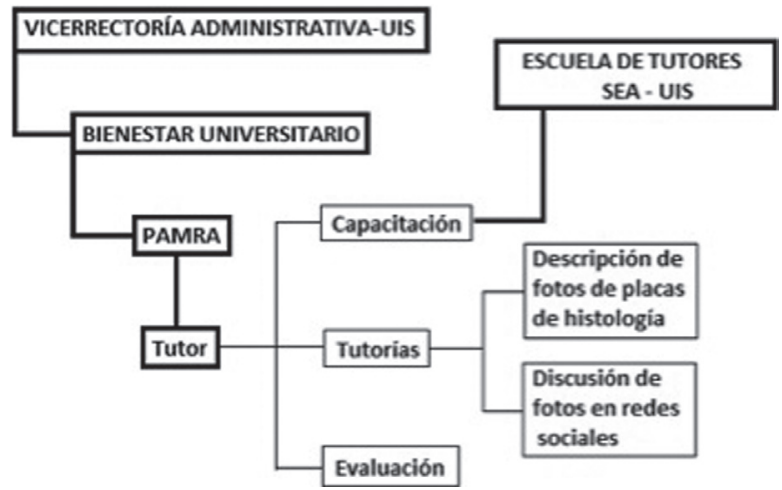

Figura. 1 Organigrama de tutorías entre pares de histología, UIS Fuente: autores.

\section{EVALUACIÓN DE LOS TUTORES}

La evaluación y el monitoreo de la actividad pedagógica que llevan a cabo los tutores se realiza según dos metodologías, reuniones y formularios. En las reuniones, las trabajadoras sociales refieren las indicaciones básicas y los comentarios acerca de los tutores, que los beneficiarios de la estrategia les transmiten de forma personal a manera de entrevista en su oficina. En la segunda metodología, 
se evalúa al tutor mediante puntuaciones obtenidas a partir de formularios que completan al menos cinco beneficiarios del grupo que maneje el tutor. Estos formularios contienen una serie de ítems como; objetivos de la tutoría, estrategia usada y despeje de dudas $^{20}$.

\section{DESCRIPCIÓN DE LA TUTORÍA DENTRO Y FUERA DEL LABORATORIO}

Las intervenciones de la tutoría se centran principalmente en dos escenarios. Dentro del laboratorio previamente a la intervención práctica se realiza una entrega de material didáctico, el cual consta de guías de identificación en las cuales se presentan las principales características que se deben encontrar en cada placa histológica de tejidos u órganos. El laboratorio de histología de la UIS cuenta con aproximadamente un total de 30 microscopios ópticos, en cada uno de ellos previamente ha sido ubicada una placa histológica por el docente encargado de la asignatura. Cada estudiante debe rotar por esos puestos visualizando las principales características de cada placa y recibiendo apoyo por parte de los docentes y tutores. Dentro de sus funciones el tutor colabora calificando exámenes y ayuda a construir los mismos (Ver Figura 2).

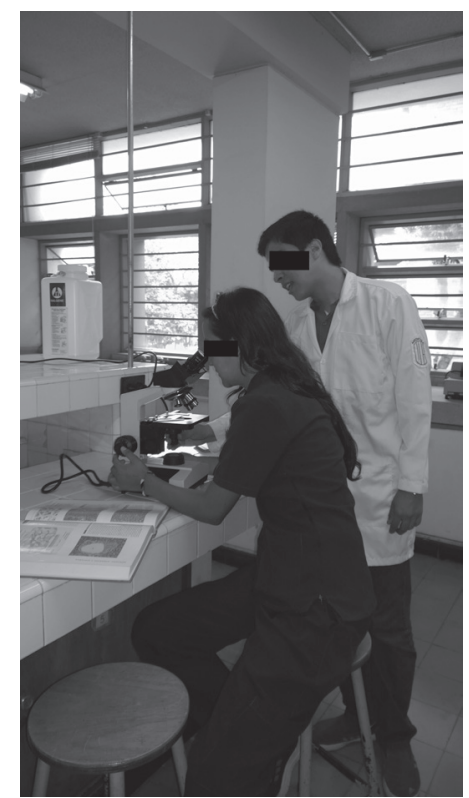

Figura 2. Tutor PAMRA-UIS asesorando en el microscopio óptico Fuente: autores.

Fuera del laboratorio por medio de redes sociales se realizan las siguientes actividades, primero un listado de los miembros que son apadrinados por el tutor; segundo se comparten fotografías de las placas histológicas del laboratorio con el fin de generar un ambiente crítico de las estructuras identificadas; y por último se informa sobre ubicación y hora de los tutoriales. Los grupos conformados pueden tener un máximo de 30 y un mínimo de 10 personas, esto dependiendo de la organización logística (Ver Figura 3).

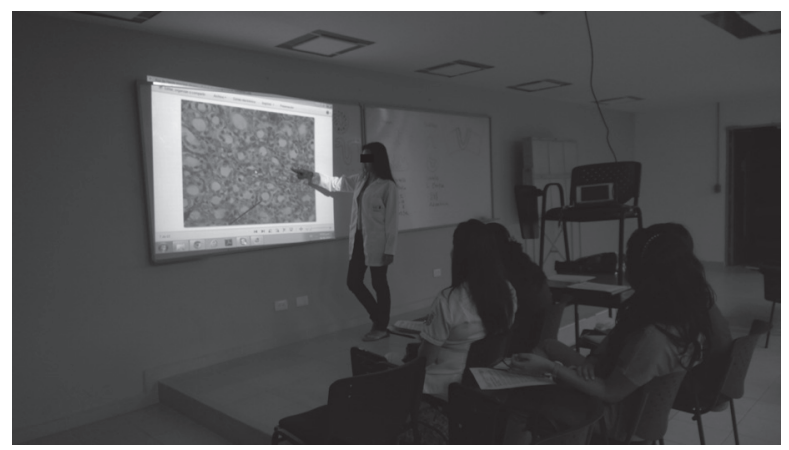

Figura 3. Tutor PAMRA-UIS en sesión fuera del laboratorio explicando imágenes de placas histológicas de riñón Fuente: autores.

Simultáneamente se realizan sesiones tutoriales presenciales. Durante estas sesiones se utilizan medios audiovisuales con video beam y computador para proyectar presentaciones previamente preparadas por el tutor en formato software Microsoft Power Point $2010^{\circledR}$. En estas presentaciones se exhiben esquemas básicos e imágenes, donde se identifican las estructuras básicas, se generan preguntas y se crea un ambiente de participación activa. Una vez cumplidos los anteriores pasos se realiza un examen, el cual consiste en 10 imágenes histológicas del tema tratado en la sesión de tutoría.

\section{CONCLUSIONES}

La tutoría entre pares es una estrategia que resulta positiva, y la importancia de suaplicación se justifica en que su implementación en instituciones universitarias ayuda a disminuir la deserción estudiantil y genera ventajas tanto para el beneficiario como para el tutor. Para los beneficiarios se mejoran sus calificaciones, refuerzan sus pre-saberes y adquieren motivaciones para estudiar, especialmente en la transición de la educación básica a la superior. Igualmente es importante para los tutores porque no solo obtienen un tipo de remuneración económica, sino que al compartir con sus compañeros adquieren valores, competencias comunicativas y una experiencia cercana a la docencia.

En la UIS se implementa esta herramienta a través 
del programa PAMRA, el cual se ha mantenido y ha crecido en la historia. Su función es hacer la convocatoria, capacitación y evaluación de los tutores, los cuales son estudiantes generalmente de semestres avanzados que participan voluntariamente. En histología los tutores asesoran al beneficiario en pequeños grupos dentro y fuera del laboratorio, asesorando en la visualización de placas histológicas y usando fotografías e imágenes para la descripción de las diferentes estructuras microscópicas.

\section{CONFLICTOS DE INTERÉS}

Los autores declaran no tener conflicto de interés.

\section{AgRADECIMIENTOS}

Agradecimientos por su colaboración en la búsqueda blibliográfica a Leidy Karina Contreras, estudiante de II nivel de Medicina de la Universidad Industrial de Santander.

\section{CONSIDERACIONES ÉtICAS}

Los participantes para las fotografías dieron su libre consentimiento informado por escrito según la Declaración de Helsinki y la resolución colombiana N8430 de 1993.

\section{REFERENCIAS BiBLIOGRÁFICAS}

1. Gómez Alicia. Enseñanza en la virtualidad. Tutorías entre pares estudiantiles y docentes. [Tesis de grado] Uruguay: Universidad de la República, facultad de humanidades y ciencias de la educación, 2013.

2. Alzate-Medina GM. Peña-Borrero LB. Peer Tutoring: Developing Writing in College Education. Univ. Psychol. 2010;9:123-38.

3. Rudland JR. Rennie SC. Medical faculty opinions of peer tutoring. Educ Health (Abingdon, Print). 2014;27(1):4-9

4. Santee J. Garavalia L. Peer Tutoring Programs in Health Professions Schools. Am. J Pharma Educ. 2006;70(3):1-10.

5. Fernández Martín FD. Arco Tirado JL. López Ortega S. Heilborn Díaz VA. Prevención del fracaso académico universitario mediante tutoría entre iguales. Rev. latinoam. Psicol. 2011;43(1):59-71.

6. Sobral DT. Cross-year peer tutoring experience in a medical school: conditions and outcomes for student tutors. Medical Educ. 2002;36:1064-70.

7. Salerno Kennedy R. Henn P. O’Flynn S. Implementing peer tutoring in a graduate medical education programme. Theclinicalteacher. 2010;7:83-9.

8. Ministerio de educación. Estadísticas de permanencia en el 2013 en Educación Superior. [Internet]. 2013 [Citad0 20 Nov 2015]. Disponible en: http://www.mineducacion.gov.co/ sistemasdeinformacion/1735/articles-254702_archivo_pdf_ estadisticas 2013.pdf

9. Castaño JJ, Cerón AC, Collazos AK, Molina AM, Osorio J, Ospina A, et al. Factores que inciden en la motivación académica en un programa de medicina, Manizales, Colombia, 2010. ArchMed. 2012;12(1):46-61.

10. Lapeña C, Sauleda N, Martínez A. Los programas institucionales de acción tutorial: una experiencia desarrollada en la universidad de alicante. RIE. 2011;29(2):341-61.

11. Cardozo CE. PAMRA reconstrucción de realidades, sueños y vidas desde una mirada colectiva. [Tesis]. Bucaramanga: Universidad Industrial de Santander. Escuela de educación, Maestría en pedagogía; 2010.

12. Laguzzi J, Bernardi S, Araujo AM, Ventura AC, Vigliano FA. Estilos de aprendizaje de los estudiantes de medicina veterinaria de la Universidad Nacional de Rosario, Argentina. Rev. electrón. vet. 2013;24(2):151-6. 